\title{
Correction to: Phase II study of weekly carboplatin in pretreated adult malignant gliomas
}

\author{
Veronica Villani ${ }^{1} \cdot$ Andrea Pace $^{1} \cdot$ Antonello Vidiri $^{2} \cdot$ Antonio Tanzilli $^{1} \cdot$ Francesca Sperati $^{3} \cdot$ Irene Terrenato $^{3}$. \\ Mariantonia Carosi ${ }^{4} \cdot$ Beatrice Casini ${ }^{4} \cdot$ Giulio Metro $^{6} \cdot$ Marta Maschio $^{1} \cdot$ Tatiana Koudriavtseva $^{1}$. \\ Francesco Cognetti ${ }^{7} \cdot$ Alessandra Fabi $^{5}$ (i)
}

Published online: 9 September 2019

๑) Springer Science+Business Media, LLC, part of Springer Nature 2019

\section{Correction to: \\ Journal of Neuro-Oncology (2019) 144:211-216 \\ https://doi.org/10.1007/s11060-019-03223-x}

In the original article, the names of authors Mariantonia Carosi and Tatiana Koudriavtseva were incorrectly captured, and author Francesco Cognetti's affiliation was incorrect. The information is correctly shown here.

Publisher's Note Springer Nature remains neutral with regard to jurisdictional claims in published maps and institutional affiliations.

The original article can be found online at https://doi.org/10.1007/ s11060-019-03223-x.

Alessandra Fabi

alessandra.fabi@ifo.gov.it

1 Neuro-Oncology Unit, IRCCS - Regina Elena National

Cancer Institute, Rome, Italy

2 Service of Neuroradiology, IRCCS - Regina Elena National

Cancer Institute, Rome, Italy

3 Biostatistic Unit, IRCCS - Regina Elena National Cancer Institute, Rome, Italy

4 Division of Neuropathology, IRCCS - Regina Elena' National Cancer Institute, Rome, Italy

5 Division of Medical Oncology, IRCCS - Regina ElenaNational Cancer Institute, Rome, Italy

6 Ospedale Santa Maria Della Misericordia Azienda Ospedaliera Di Perugia, Perugia, Italy

7 Università La Sapienza Roma, Rome, Italy 